\title{
Spectrum-splitting photovoltaic system using bifacial cells for high energy yield
}

Chrysler, Benjamin, Tan, Xuessen, Zhao, Jianbo, Kostuk, Raymond

Benjamin D. Chrysler, Xuessen Tan, Jianbo Zhao, Raymond K. Kostuk, "Spectrum-splitting photovoltaic system using bifacial cells for high energy yield," Proc. SPIE 11121, New Concepts in Solar and Thermal Radiation Conversion II, 111210B (9 September 2019); doi: 10.1117/12.2528049

EDent: SPIE Optical Engineering + Applications, 2019, San Diego, California, United States 


\title{
Spectrum-Splitting Photovoltaic System using Bifacial Cells for High Energy Yield
}

\author{
Benjamin D. Chrysler ${ }^{*}$, Xuessen Tan ${ }^{\mathrm{b}}$, Jianbo Zhao ${ }^{\mathrm{b}}$, Raymond K. Kostuk ${ }^{\mathrm{a}, \mathrm{b}}$ \\ ${ }^{A}$ University of Arizona, College of Optical Sciences, 1630 E. University Blvd., Tucson, Arizona \\ 85721, USA; ${ }^{\mathrm{B}}$ University of Arizona, Department of Electrical and Computer Engineering, $1230 \mathrm{E}$. \\ Speedway Blvd. 1230 E. Speedway Blvd., Tucson, Arizona, 85721, USA
}

\begin{abstract}
Keywords: Spectrum-Splitting, Holography, Diffraction, Photovoltaics, Bifacial Cells, Energy Conversion

In this paper a spectrum-splitting photovoltaic system is proposed that uses bifacial silicon solar cells to maximize total energy yield. The system is unique in its ability to convert direct sunlight with high-efficiency $(>30 \%)$ while simultaneously converting diffuse and rear-side irradiance. A volume holographic lens array is used to divide the solar spectrum into spectral bands optimized for conversion by wide-bandgap and bifacial silicon solar cells. An approach for simulating the energy yield, optimizing the holographic lens array, and analyzing the effect of concentration ratio, aspect ratio, and illumination characteristics is described. Design examples for two different solar cell combinations are provided. A GaAs and bifacial silicon combination achieves an energy conversion efficiency of $32.0 \%$ and a MgCdTe and bifacial silicon combination achieves a $31.0 \%$ energy conversion efficiency. Additional solutions are provided when constraints on concentration ratio and aspect ratio are applied, allowing the designer to balance energy yield with cost and size considerations. The performance of the proposed system is compared to conventional monofacial silicon, bifacial silicon, and monofacial spectrum-splitting modules, and show that improvements in energy yield of over $45 \%, 25 \%$, and $10 \%$ can be achieved, respectively.
\end{abstract}

\section{INTRODUCTION}

The Levelized Cost of Energy (LCOE) of Photovoltaic (PV) solar energy has fallen significantly over the past few decades. The reduction in LCOE is primarily due to a lower price of silicon wafers and from an increase in the efficiency of silicon solar cells. In the next decade the LCOE of solar energy is only expected to decrease by $15 \%$ in comparison to $80 \%$ in the previous decade ${ }^{1}$. The stabilization in the LCOE of solar energy is due to a maturing wafer manufacturing industry and relatively small expected increases in the efficiency of silicon solar cells which have already achieved $92 \%$ of their theoretical limit ${ }^{2}$. In order to significantly reduce the LCOE of solar energy beyond what is projected it will be necessary to use new techniques that have higher energy yield than conventional silicon modules but have low component and manufacturing costs.

Several systems have been explored for increasing the energy yield of photovoltaic modules. One extensively studied system uses high concentration optics to focus sunlight onto small, high-efficiency multi-junction solar cells (CPV). While CPV systems achieve high efficiency under direct sunlight, they fail to perform as expected in realistic solar conditions ${ }^{3}$. While there are several reasons for the lower than expected performance, a key reason is the inability to convert diffuse sunlight, which consists of approximately $20-40 \%$ of total incident sunlight ${ }^{4}$ even in predominately sunny regions, such as Tucson, Arizona. Another disadvantage of CPV systems is that they require expensive and bulky tracking systems and are not compatible with conventional one-axis tracking systems used in many utility-scale PV power plants.

Many of the disadvantages of CPV systems are alleviated in Spectrum-Splitting Photovoltaic (SSPV) systems. Instead of using small, monolithically integrated multijunction cells, SSPV systems use a set of independently connected singlejunction solar cells in combination with optical filters that separate incident sunlight into spectral bands ${ }^{5-7}$. Each spectral band is redirected to a PV cell that has an optimal bandgap-energy and spectral response for converting that portion of the

New Concepts in Solar and Thermal Radiation Conversion II, edited by Jeremy N. Munday, Peter Bermel, Proc. of SPIE Vol. 11121, 111210B · C 2019 SPIE · CCC code: 0277-786X/19/\$21 · doi: 10.1117/12.2528049 
spectrum. Many promising SSPV system designs are based on the use of volume holographic optical elements ${ }^{8-9}$ and can be designed in ways that yield Power Conversion Efficiencies (PCE) greater than 30\%, convert diffuse sunlight, and have form-factors comparable to conventional silicon modules.

Another class of PV system that has higher energy yield are Bifacial Photovoltaic (BFPV) modules ${ }^{10}$. Bifacial systems have become more common in recent years and it is expected that they will have a $30 \%$ market share by $2025^{11}$. BPV modules use bifacial solar cells which are made of silicon and have PN-junctions and contact grid patterns optimized for converting light from both the front and rear sides of the cell. While the technique does not improve the PCE of an individual cell surface, the energy yield of the module is improved by 10-50\% depending on the surface albedo and PV module array configuration ${ }^{12-15}$.

In this paper, a Bifacial Spectrum-Splitting Photovoltaic (BF-SSPV) system is proposed that combines the concepts of SSPV and BFPV systems. The system converts direct sunlight at high efficiency (>30\%), converts diffuse and rear-side irradiance, and can be designed in packages comparable to conventional flat-panel silicon modules. The system architecture is shown in Fig. 1 and consists of a volume holographic lens array positioned over alternating strips of widebandgap cells and bifacial silicon cells. Spectrum-splitting is accomplished by the holographic element. The holographic element provides a sharp spectral filter cutoff by focusing light at the bandgap wavelength of the wide-bandgap cell to the opposing boundary between the PV cells. The holographic element provides high optical efficiency by setting the Bragg condition to efficiently diffract wavelengths in the center of each spectral band. Diffuse sunlight is converted since light at non-normal incidence angles do not meet the Bragg-condition of the hologram and are transmitted without diffraction onto the PV cell surface.

While spectrum-splitting is accomplished in a similar way to the design provided by Vorndran et. $\mathrm{al}^{9}$, the BF-SSPV system has the unique capability of converting rear-side irradiance by using bifacial silicon cells and transparent glass on the rearside of the module. Rear-side irradiance is converted when light reflected from the ground surface is transmitted through the rear glass and converted by the bifacial silicon cells. The rear-side energy yield is enhanced by increasing the concentration on the monofacial wide-bandgap cell or by using a diffusing scattering surface on the rear-side of the widebandgap cell to re-direct light through total internal reflection onto the bifacial silicon cell.

In this paper we provide a technique for simulating the energy yield of the BF-SSPV system and an optimization technique for the volume holographic lens array. We then analyze the effect of key system design parameters on the overall energy yield in order to balance design tradeoffs. Important geometrical parameters are width of the wide-bandgap cell, $W_{W B G}$, the width of the bifacial silicon cell, $W_{s i}$, the thickness of the front encapsulating material, $H_{\text {front }}$, and the thickness of the rear encapsulating material, $H_{\text {rear }}$. These parameters can be expressed in terms of the concentration ratio, $C R=\frac{W_{W B G}+W_{S i}}{W_{W B G}}$, the front aspect ratio, $A=\frac{H_{\text {front }}}{W_{W B G}+W_{s i}}$, and the rear aspect ratio, $A_{\text {rear }}=\frac{H_{\text {rear }}}{W_{W B G}+W_{s i}}$. These parameters studied since they are related to practical considerations such as the cost of the component PV cells and the size and weight of the module. Higher concentration ratio and lower aspect ratio modules may be more commercially feasible due to their compact packaging and reduced use of expensive wide-bandgap PV cells, even though they have lower total energy yield. 


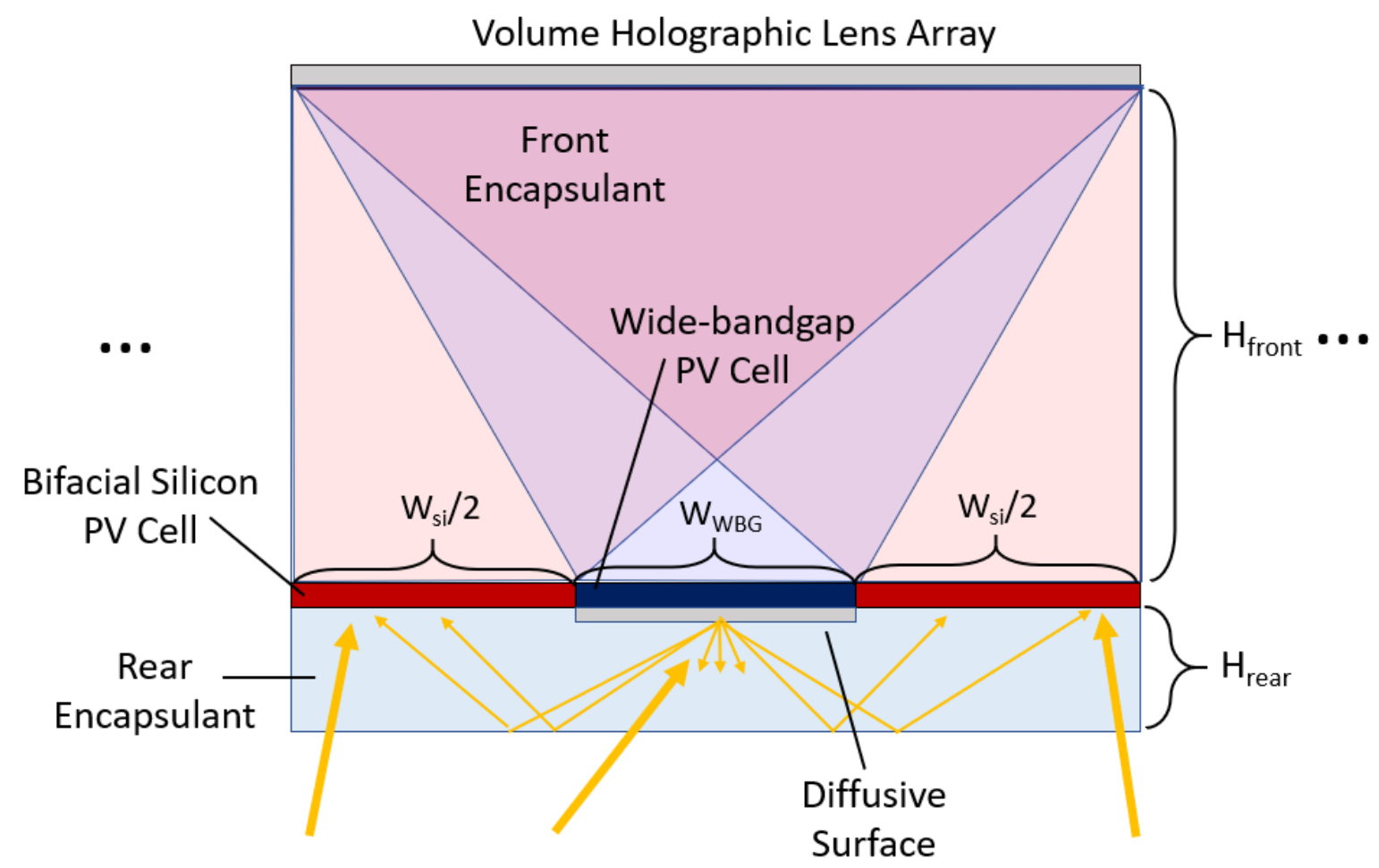

Figure 1: Illustration of a repeating "unit cell" in the bifacial spectrum-splitting photovoltaic system. Direct sunlight is focused and dispersed by a volume holographic lens array onto alternating strips of bifacial silicon and wide-bandgap PV cells. Rear-side irradiation is converted by the bifacial solar cells. The rear-side of the wide-bandgap cell is coated with a diffuse scattering surface to redirect some of the irradiation onto the bifacial silicon through total internal reflection.

\section{ENERGY YIELD OPTIMIZATION}

In this section we provide a technique for optimizing the holographic element of the BF-SSPV system. The goal of the optimization is to maximize the PCE of the BF-SSPV system and it is assumed that the aspect ratio, concentration ratio, and PV cell conversion characteristics are given. The hologram parameters to be optimized are the K-vector, index modulation, and film thickness. The optimization is described in a set of four processes. In process 'i' the grating K-vector is optimized based on the geometry of the system and the spectral bands of the PV cells. In process 'ii' the diffraction efficiency is maximized by tuning the index modulation. In process 'iii' the energy yield and the PCE are calculated. In process 'iv' the holographic film thickness is optimized. Since the optimal index modulation is dependent on the holographic film thickness, process 'ii' must be repeated for each iteration of the film thickness optimization. Process 'iii' is also repeated for each iteration of the film thickness optimization and the PCE is used as the figure of merit. Unlike previous hologram design processes for solar applications ${ }^{9,16}$, the optimization is not constrained by the Kogelnik Qparameter, which limits the hologram to diffract into a single order, but instead the optimization uses Rigorous Coupled Wave Analysis to simulate multiple diffraction orders and directly maximizes the PCE regardless of the number of diffraction orders generated.

After the holographic element is optimized, the effect of variables such as concentration ratio, aspect ratio, and illumination can be analyzed and used to design a variety of systems that meet different constraints. 


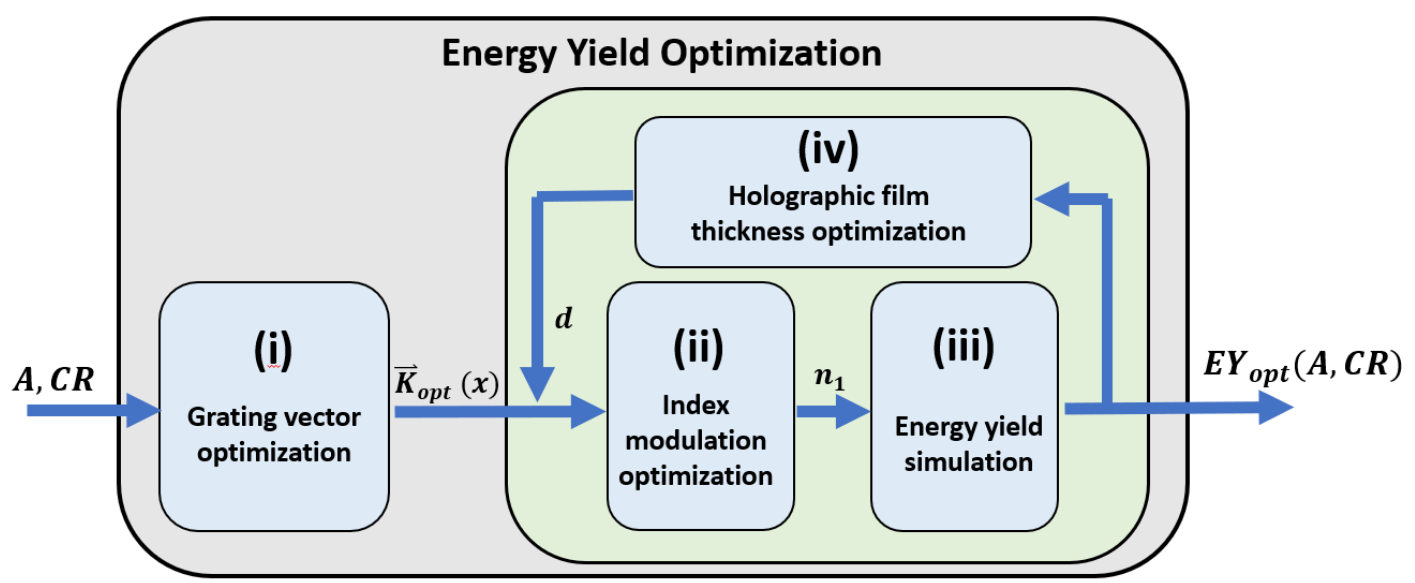

Figure 2: Block diagram of the four-step energy yield optimization process. For a given aspect ratio, $A$, and concentration ratio, $C R$, the optimizer returns the optimum energy yield $E Y_{o p t}(A, C R)$ by optimizing the grating vector, $\vec{K}_{o p t}(x)$, the index modulation, $n_{1}$, and the holographic film thickness, $d$.

\subsection{Grating Vector Optimization}

In process ' $i$ ' the optimal grating K-vector of the holographic element, $\vec{K}_{o p t}(x)$, is determined based on the system geometry similarly to references ${ }^{9,17}$. In this method the desired K-vector, $\vec{K}_{\text {target }}(x)$, diffracts and focuses light at a "transition" wavelength, equal to the bandgap wavelength of the wide-bandgap cell, to the boundary between the two PV cells. The Bragg condition is set so that normally incident light is diffracted at highest efficiency in the center of the spectral band of the PV cell it is diffracting to. Unfortunately, these conditions cannot simultaneously be fulfilled along the entire aperture of the holographic element using a standard two-point-source construction method with point sources located at $\overrightarrow{r_{1}}$ and $\overrightarrow{r_{2}}$. To find the best approximation of $\vec{K}_{\text {target }}(x)$, the error between the target grating vector and the grating vector constructed with point sources located at $\overrightarrow{r_{1}}$ and $\overrightarrow{r_{2}}, \vec{K}\left(x, \overrightarrow{r_{1}}, \overrightarrow{r_{2}}\right)$, is minimized:

$$
\operatorname{minimize}_{\overrightarrow{r_{1}}, \overrightarrow{r_{2}}}\left\{\operatorname{abs}\left(\vec{K}\left(x, \overrightarrow{r_{1}}, \overrightarrow{r_{2}}\right)-\vec{K}_{\text {target }}(x)\right)\right\}
$$

Where the minimize function finds the point sources $\vec{r}_{1, o p t}$ and $\vec{r}_{2, o p t}$ that minimize the error vector in the argument. Each component of the error vector is minimized by the finding least squares sum using the optimized least squares function in Python. Consequently, both the lateral and longitudinal components of the K-vector are optimized. After determining the optimal point sources the optimal K-vector is constructed:

$$
\vec{K}_{o p t}(x)=\vec{K}\left(x, \vec{r}_{1, o p t}, \vec{r}_{2, o p t}\right)
$$

\subsection{Index Modulation Optimization}

In process ' $\mathrm{ii}$ ' we optimize the index modulation, $n_{1}$, given $\vec{K}_{o p t}(x)$ provided in process ' $\mathrm{i}$ ' and the holographic film thickness, $d$. The index modulation is optimized by scanning through different values of $n_{1}$ for both holographic lenses, simulating the diffraction efficiency for the Bragg-matched wavelength, and choosing the value that maximizes the diffraction efficiency. The index modulation is restricted to a range that is attainable for the holographic material in consideration. In our optimization, we assume dichromated gelatin ${ }^{18}$, which can obtain index modulation up to 0.1 .

\subsection{Energy Yield Simulation}

In process 'iii' the total energy yield, $E Y_{\text {total }}$, of the B-SSPV system is determined based on the properties of the solar illumination, holographic element, and component PV cells. A set of equations and simulations are used to determine the direct, diffuse, and rear-side illumination components of the energy yield, represented as $E Y_{\text {direct }}, E Y_{\text {diffuse }}, E Y_{\text {rear }}$ respectively. The total energy yield is the sum of the individual components: 


$$
E Y_{\text {total }}=E Y_{\text {direct }}+E Y_{\text {diffuse }}+E Y_{\text {rear }}
$$

The first step in obtaining the direct component is simulating the diffraction of normally incident sunlight onto the solar cells. Given holographic film thickness, $d$, and holographic lens index modulation, $n_{1}$, the spectral diffraction efficiency is determined at a given position along the element using Rigorous Coupled Wave Analysis (RCWA) coded in Python ${ }^{19}$ for five total transmission and reflection orders. The K-vector of the holographic element is sampled in 80 positions across the entire aperture of the holographic element and simulated at each position to obtain the spectral diffraction efficiency, $\eta_{i}(x, \lambda)$, as a function of position across the aperture of the holographic element. The number of sampling positions was determined to provide sufficient resolution for the variation in spectral diffraction efficiency across the element.

After calculating the diffraction efficiency of the holographic element, the illumination of the PV cells can be modeled using FRED non-sequential raytracing ${ }^{20}$. First, a model for the holographic element and the PV cells is setup with the desired aspect and concentration ratios. An evenly-weighted broadband source is situated over the holographic element. The K-vector and spectral diffraction efficiency are entered for each sampled position across the aperture of the holographic element. Next, the illumination is modeled using a Monte-Carlo raytrace simulation and is analyzed for each PV cell using the Spectral Analyses functions in FRED. Since the spectral source is evenly weighted, the Spectral Analyses determines the fraction of light at each wavelength that is collected by each PV cell. This parameter is called the Spectral Optical Efficiency $\left(\operatorname{SOE}_{i}(\lambda)\right)$ :

$$
\operatorname{SOE}_{i}(\lambda)=\frac{E_{i}(\lambda)}{G(\lambda)}
$$

Where $E_{i}(\lambda)$ is the spectral irradiance on the $\mathrm{i}^{\text {th }} \mathrm{PV}$ cell and $G(\lambda)$ is the spectral irradiance incident on the unit cell.

The Power Conversion Efficiency $(P C E)$ of the system for direct sunlight can be determined using the $S O E_{i}(\lambda)$ provided by the raytracing simulation, the Spectral Conversion Efficiency $\left(S C E_{i}(\lambda)\right)$ of each PV cell, and the incident direct illumination spectrum, $A M 1.5_{D}(\lambda)^{6}$ :

$$
P C E=\sum_{i} \frac{\int A M 1.5_{D}(\lambda) \cdot S O E_{i}(\lambda) \cdot S C E_{i}(\lambda) \cdot \mathrm{d} \lambda}{\int A M 1.5_{D}(\lambda) \cdot d \lambda}
$$

The energy yield from direct sunlight is determined using direct normal solar insolation data, $E_{\text {direct }}$, obtained from the NREL TMY3 database ${ }^{4}$ :

$$
E Y_{\text {direct }}=P C E \cdot E_{\text {direct }}
$$

Diffuse energy yield is estimated by weighting the incident diffuse energy, $E_{\text {diffuse }}$, by the fractional area each PV cell fills the unit cell area and the individual conversion efficiencies of the PV cells, $\eta_{W B G}$ and $\eta_{\text {silicon }}$ :

$$
E Y_{\text {diffuse }}=\left(\frac{1}{C R} \cdot \eta_{W B G}+\left[1-\frac{1}{C R}\right] \cdot \eta_{\text {silicon }}\right) \cdot E_{\text {diffuse }}
$$

The rear-side energy yield is obtained assuming that the incident rear-side energy, $E_{\text {rear }}$, is related to the total direct and diffuse energy by the rear irradiance factor ${ }^{10}, \chi=\frac{E_{\text {rear }}}{E_{\text {direct }}+E_{\text {diffuse }}}$ and weighting the rear-side energy by the efficiency and fractional area of the bifacial silicon cell:

$$
E Y_{\text {rear }}=\left(1-\frac{1}{C R}\right) \cdot \eta_{s i} \cdot \chi \cdot\left(E_{\text {direct }}+E_{\text {diffuse }}\right)
$$

When a diffusing surface is added to the rear-side of the wide-bandgap cell, an additional simulation is performed to find the fraction of light incident on the bifacial silicon, $f\left(C R, A_{\text {rear }}\right)$, where $A_{\text {rear }}=\frac{H_{\text {rear }}}{W_{W B G}+W_{s i}}$ is the rear aspect ratio of the system. This simulation is also performed in FRED. In this model, the PV cells are laid out similarly to the front-side simulation, with cell widths determined by $C$. A Lambertian scattering surface is placed on the rear-side of the widebandgap cell and an encapsulating glass with $n=1.5$ with a thickness determined by $A_{\text {rear }}$ is placed underneath the unit cell. Rays are traced from the bottom aperture of the system and the fraction of rays incident on the bifacial silicon is 
$f\left(C R, A_{\text {rear }}\right)$. When using a diffuser, the rear-side energy yield can be determined by replacing the fraction of area covered by silicon with $f\left(C R, A_{\text {rear }}\right)$ :

$$
E Y_{\text {rear }}=f\left(C R, A_{\text {rear }}\right) \cdot \eta_{\text {si }} \cdot \chi \cdot\left(E_{\text {direct }}+E_{\text {diffuse }}\right)
$$

\subsection{Holographic Film Thickness Optimization}

In process 'iv' the holographic film thickness is optimized by scanning through a range of film thickness values that can be fabricated in dichromated gelatin. In this simulation, film thicknesses were assumed to lie between 1um and 30um. Since the optimal index modulation is dependent on the film thickness, the the index modulation is optimized using process 'ii' for each film thickness value. The $P C E$ for each film thickness value is determined using process 'iii' and the value that gives the highest PCE is selected as the optimal film thickness. After determining the optimal thickness, the algorithm returns the optimal energy yield, $E Y_{\text {opt }}(A, C R)$.

\section{DESIGN EXAMPLES}

In this section, two design examples are provided for different wide-bandgap PV cell types. First the light collection enhancement of the rear-diffuser is modeled and then the energy yield performance of the BF-SSPV system is modeled.

The rear-diffuser was modeled using the method described at the end of Section 2.3. In FRED, a 96\% reflecting Lambertian scattering surface was placed on the rear side of the wide-bandgap solar cell and the solar cells were encapsulated in a rear glass with a refractive index of $\mathrm{n}=1.5$. The dimensions of the solar cells and rear glass are determined based on $C R$ and $A_{\text {rear }}$. The fraction of light collected with and without the diffuser, $f\left(C R, A_{\text {rear }}\right)$, is shown in Figs. 3a,b. The results show the benefit of the diffuser is greatest for concentrations around $C R \approx 2$ where rear-side energy collection increases by up to $25 \%$. The best results are obtained when $A_{\text {rear }} \approx 0.1-0.2$, which allows an angular range sufficient for light to be reflected by total internal reflection and redirected to the bifacial silicon. There is a slight decrease in collection efficiency for higher $A_{\text {rear }}$ because it is more likely that light will scatter to a diffuser in a neighboring unit cell and subsequently be scattered out of the escape cone. Based on these results, we assumed that $A_{\text {rear }}=0.2$ in the following examples.
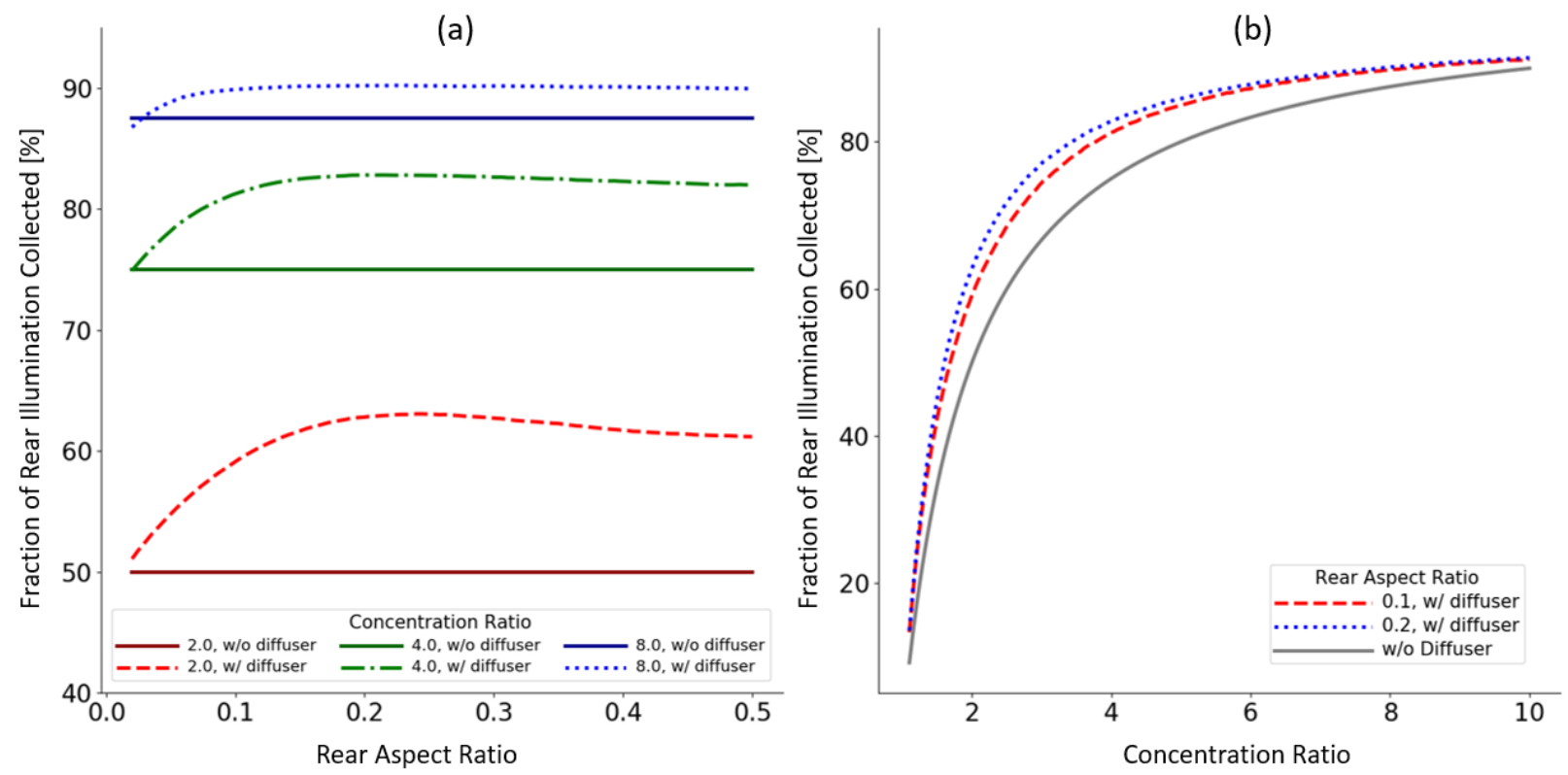

Figure 3: Fraction of rear-side illumination collected, $f\left(C \mathrm{R}, A_{\text {rear }}\right)$, with and without a diffuser as a function of (a) rear aspect ratio, $A_{\text {rear }}$, and (b) concentration, $C$ R.

In the first example we use a $28.8 \%$ efficient Alta GaAs cell ${ }^{21}$ with a bandgap energy of $1.4 \mathrm{eV}$ and in the second example we use $21.1 \%$ efficient $\mathrm{MgCdTe}$ cell ${ }^{22}$ with a bandgap energy of $1.7 \mathrm{eV}$. All examples use $22.5 \%$ efficient bifacial silicon 
that is based on Prism Solar Technology's n-type bifacial cells ${ }^{23}$. For these design examples, we report system performance using the Energy Conversion Efficiency (ECE) metric which we define as the ratio of the total energy yield to the total incident direct and diffuse energy:

$$
E C E=\frac{E Y_{\text {total }}}{E_{\text {direct }}+E_{\text {diffuse }}}
$$

The system was simulated assuming a ratio of diffuse to total front-side energy, $\frac{E_{\text {diffuse }}}{E_{\text {diffuse }}+E_{\text {direct }}}=10 \%$, which is similar to the AM1.5G standard. The rear irradiance factor, $\chi=15 \%$, was selected as a typical value for utility-scale PV power plants as predicted by several models ${ }^{10-14}$.

The optimized results for GaAs are shown in Figs. 4a,b and the results for MgCdTe are shown in Figs. 4c,d. All the results are summarized in Table 1. The results show a maximum ECE of 32.9\% for GaAs and 31.0\% for MgCdTe. Concentration ratios of $C R \approx 3$ and aspect ratios $A \approx 1.0-1.5$ tended to yield the highest ECE. Higher ECE is obtained assuming $\chi=$ $30 \%$, which is a typical value for PV modules arrays with higher surface albedo, less tightly packed spacing, and greater distance from the ground. In this scenario, the GaAs system obtains ECE $=35.0 \%$ and the MgCdTe system obtains ECE $=33.6 \%$. Lower efficiencies are obtained for higher concentrations and lower aspect ratios, but can still exceed 30\%. For example, GaAs achieves $\mathrm{ECE}=30.3 \%$ at a concentration of $8 \mathrm{X}$ and $\mathrm{ECE}=32.1 \%$ for an aspect ratio of 0.5 . These results indicate that high energy yield BF-SSPV systems can be designed to meet a variety of design constraints.

Table 1: Energy Conversion Efficiency for different system configurations and illuminations conditions.

\begin{tabular}{|c|c|c|c|c|c|c|c|c|}
\hline & \multicolumn{3}{|c|}{ GaAs } & \multicolumn{3}{|c|}{ MgCdTe } \\
\hline & & & $\begin{array}{l}\text { Highest } \\
\text { ECE }\end{array}$ & $\mathrm{CR}=8 \mathrm{x}$ & $\mathrm{A}=0.5$ & $\begin{array}{l}\text { Highest } \\
\text { ECE }\end{array}$ & $\mathrm{CR}=8 \mathrm{x}$ & $A=0.5$ \\
\hline \multirow{4}{*}{$\begin{array}{c}\text { Energy } \\
\text { Conversion } \\
\text { Efficiency }\end{array}$} & \multirow{2}{*}{$\begin{array}{l}\chi= \\
15 \%\end{array}$} & $\begin{array}{c}\text { w/o } \\
\text { diffuser }\end{array}$ & $32.1 \%$ & $30.1 \%$ & $31.4 \%$ & $30.4 \%$ & $28.9 \%$ & $28.9 \%$ \\
\hline & & $\begin{array}{c}\text { w/ } \\
\text { diffuser }\end{array}$ & $32.9 \%$ & $30.3 \%$ & $32.1 \%$ & $31.0 \%$ & $29.1 \%$ & $29.6 \%$ \\
\hline & \multirow{2}{*}{$\begin{array}{c}\chi= \\
30 \%\end{array}$} & $\begin{array}{c}\text { w/o } \\
\text { diffuser }\end{array}$ & $33.9 \%$ & $33.1 \%$ & $33.2 \%$ & $32.7 \%$ & $31.9 \%$ & $31.2 \%$ \\
\hline & & $\begin{array}{c}\mathrm{w} / \\
\text { diffuser }\end{array}$ & $35.0 \%$ & $33.3 \%$ & $34.2 \%$ & $33.6 \%$ & $32.1 \%$ & $32.1 \%$ \\
\hline
\end{tabular}


(a)
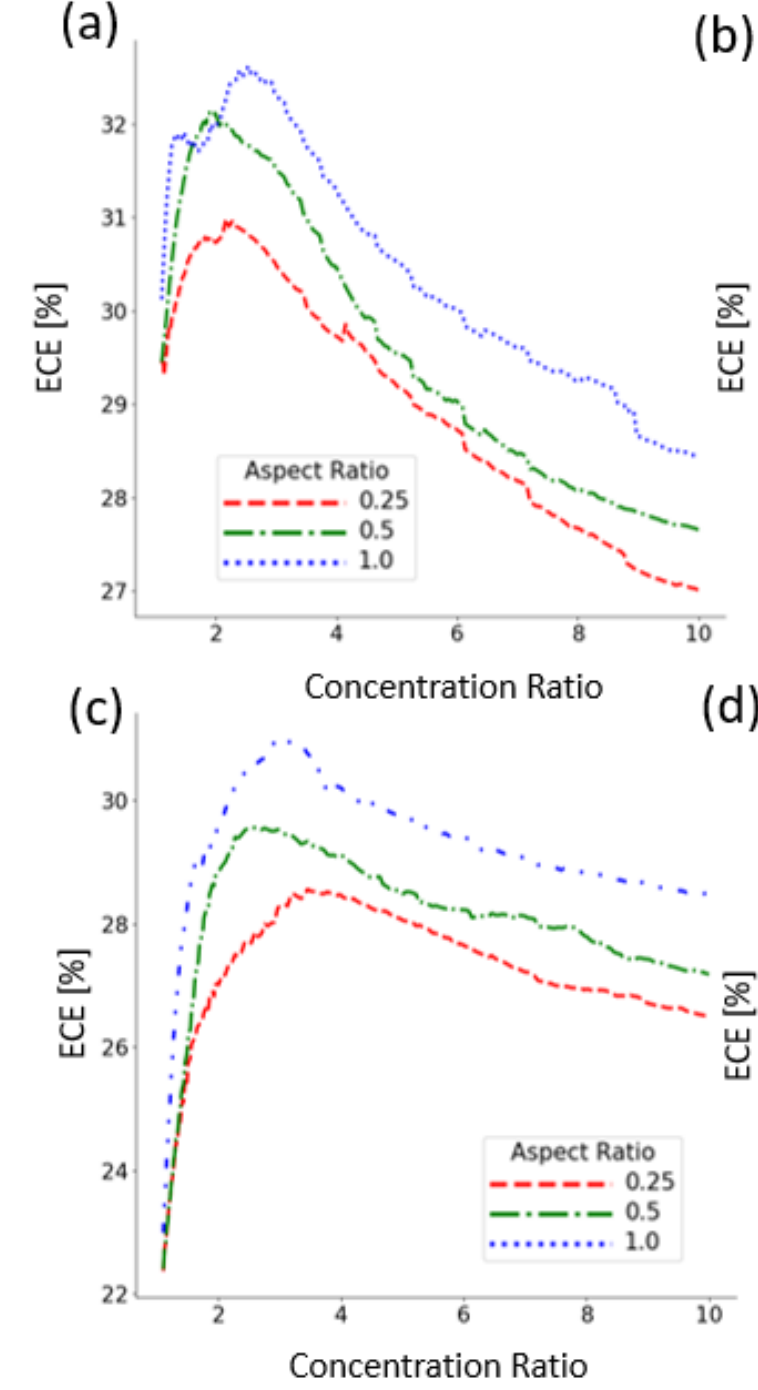

(b)
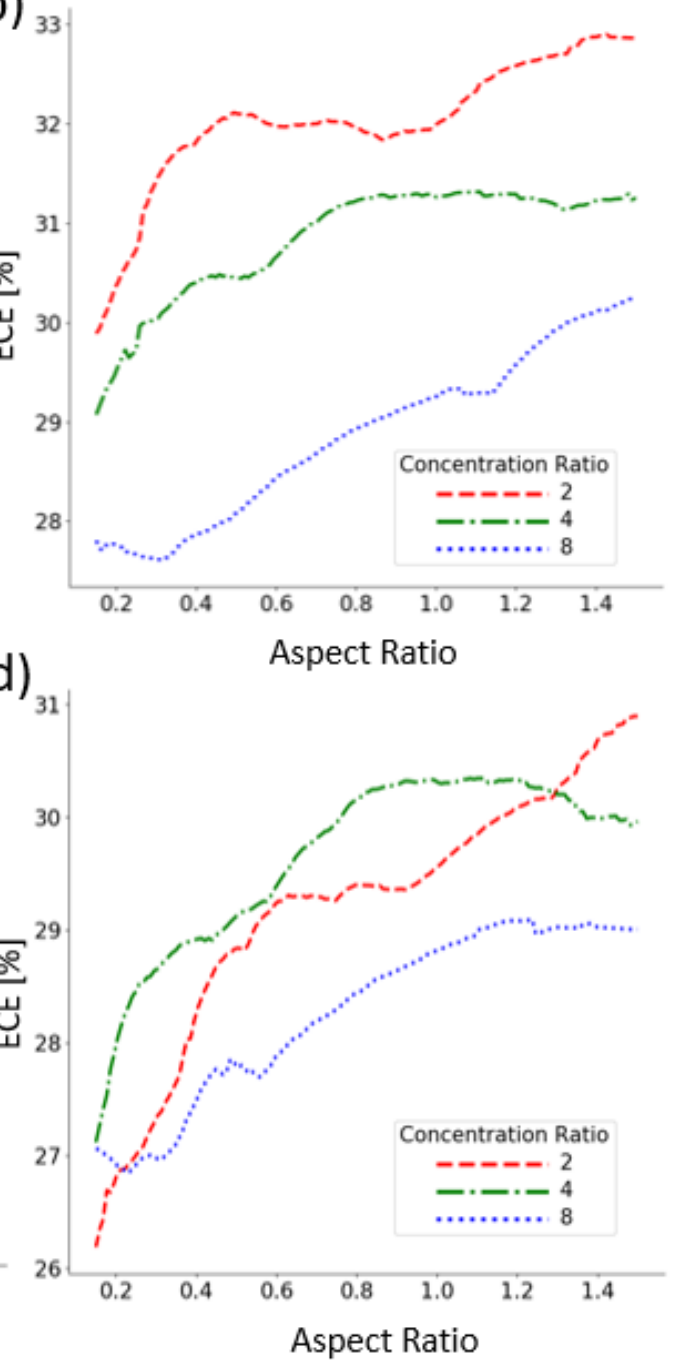

Figure 4: Simulated Energy Conversion Efficiency of the proposed bifacial spectrum-splitting system with $\chi=15 \%$ for different system parameters: (a) GaAs wide-bandgap cell plotted as a function of concentration ratio, (b) GaAs wide-bandgap cell plotted as a function of aspect ratio, (c) $\mathrm{MgCdTe}$ wide-bandgap cell plotted as a function of concentration ratio, (d) MgCdTe wide-bandgap cell plotted as a function aspect ratio.

\section{COMPARISON WITH OTHER PHOTOVOLTAIC SYSTEMS}

In this section we compare the energy yield performance of the BF-SSPV system to other photovoltaic systems. The simulation results in this section are taken from the best performing configurations presented in Section 3 . The total yearly energy yield of BF-SSPV, monofacial-SSPV, bifacial silicon, and monofacial silicon modules are presented in Figs. 5a,b as a function of the irradiance factor, $\chi$. Results are presented for systems based on both GaAs and MgCdTe. The simulation results use direct and diffuse yearly insolation values taken from TMY3 for Tucson and Seattle ${ }^{4}$. While the absolute energy yield is lower Seattle where there is a larger fraction of diffuse sunlight (33.5\%) compared to Tucson (17.0\%), in both locations the BF-SSPV systems have higher energy yield than equivalent monofacial-SSPV, monofacial silicon, and bifacial silicon modules. The results are summarized in Table 2 as the percent improvement in energy yield of the BFSSPV system compared to other PV systems. The results indicate that when $\chi=15 \%$ the improvement in energy yield can exceed $10 \%$ compared to a monofacial SSPV system, $25 \%$ compared to a bifacial silicon module, and $45 \%$ compared to a monofacial silicon module. 


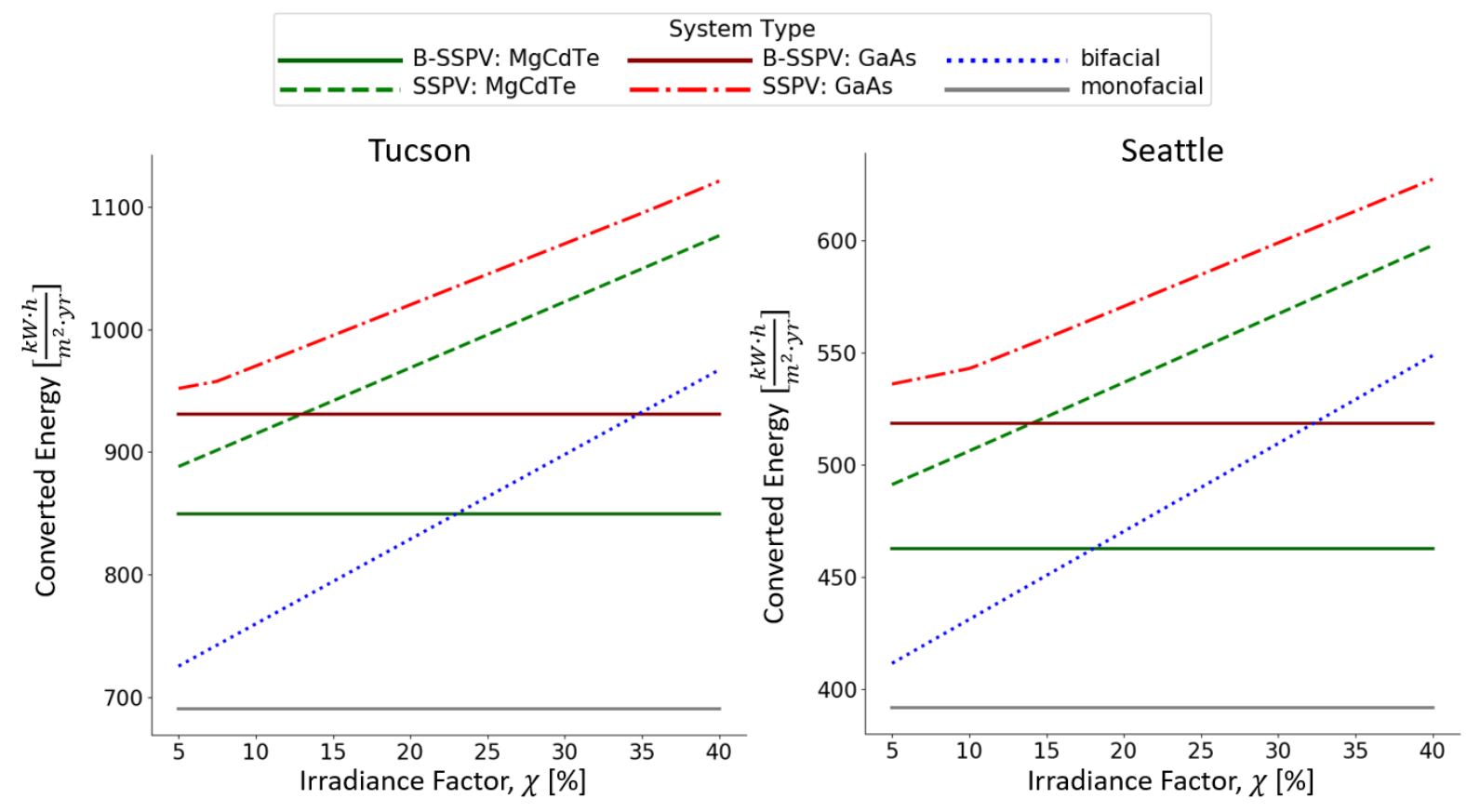

Figure 5: Yearly energy yield of different photovoltaic systems as a function of irradiance factor, $\chi$, in (a) Tucson and (b) Seattle.

Table 2: Improvement in Energy Yield compared to three types of PV systems: monofacial spectrum-splitting, bifacial silicon, and monofacial silicon. The simulation data is assuming Tucson's yearly direct and diffuse insolation and equivalent PV cell types are assumed for the comparisons.

\begin{tabular}{|c|c|c|c|c|}
\cline { 2 - 5 } \multicolumn{1}{c|}{} & \multicolumn{2}{c|}{ GaAs } & \multicolumn{2}{c|}{ MgCdTe } \\
\cline { 2 - 5 } \multicolumn{1}{c|}{} & $\chi=15 \%$ & $\chi=30 \%$ & $\chi=15 \%$ & $\chi=30 \%$ \\
\hline $\begin{array}{c}\text { Monfacial } \\
\text { Spectrum- } \\
\text { Splitting }\end{array}$ & $7.8 \%$ & $14.8 \%$ & $10.5 \%$ & $18.7 \%$ \\
\hline $\begin{array}{c}\text { Bifacial } \\
\text { Silicon }\end{array}$ & $26.4 \%$ & $19.0 \%$ & $18.1 \%$ & $12.3 \%$ \\
\hline $\begin{array}{c}\text { Monofacial } \\
\text { Silicon }\end{array}$ & $45.3 \%$ & $54.7 \%$ & $35.9 \%$ & $46.0 \%$ \\
\hline
\end{tabular}

\section{CONCLUSION}

In this paper we propose a photovoltaic system that achieves high energy yield by combining the concepts of volume holographic spectrum-splitting and bifacial conversion. Direct sunlight is converted at high efficiency by an array of volume holographic lenses that focus and disperse light onto alternating wide-bandgap and narrow-bandgap bifacial silicon photovoltaic cells. Diffuse sunlight is transmitted through the holographic element and converted, while rear-side illumination is partially collected by the bifacial silicon cells. A diffuser placed on the rear-side of the wide-bandgap cell increases rear-side energy conversion by up to $25 \%$. A simulation and optimization algorithm is presented that allows for efficient and systematic study of the design space for holographic spectrum-splitting systems. The results for systems using $28.8 \%$ efficient GaAs and $21.1 \%$ efficient MgCdTe wide-bandgap cells combined with $22.5 \%$ efficient bifacial silicon cells are presented. With the aid of a rear diffuser, the Energy Conversion Efficiency of the system is $32.9 \%$ for GaAs and $31.0 \%$ for $\mathrm{MgCdTe}$ using a typical rear-side irradiance for utility-scale installations. Results are presented for different 
concentration ratios and aspect ratios so that the system designer can balance factors such as cost of the wide-bandgap material, compactness of the system, and total energy yield. The proposed system is also compared to other photovoltaic systems and it is found the design can provide $10 \%, 25 \%$, and $45 \%$ higher energy yield compared to monofacial spectrumsplitting, bifacial silicon, and monofacial silicon PV modules respectively.

\section{ACKNOWLEDGEMENTS}

We would like to acknowledge support from our sponsors: NSF/DOE ERC cooperative agreement No. EEC-1041895 and NSF grant ECCS-1405619. Ben Chrysler would like to acknowledge support from the National Science Foundation Graduate Research Fellowship Program Grant (DGE-1143953). Any opinions, findings, and conclusions or recommendations expressed in this material are those of the authors and do not necessarily reflect the views of the National Science Foundation.

\section{REFERENCES}

[1] Woodhouse, M. A., Smith, B., Ramdas A., Margolis R.M., "Crystalline Silicon Photovoltaic Module Manufacturing Costs and Sustainable Pricing: 1H 2018 Benchmark and Cost Reduction Road Map," National Renewable Energy Lab, Golden, CO, No. NREL/TP-6A20-72134 (2019).

[2] Schindler, F., Fell, A., Müller, R., Benick, J., Richter, A., Feldmann, F., Krenckel, P., Riepe, A., Schubert, M.C., Glunz, S.W., "Towards the efficiency limits of multicrystalline silicon solar cells," Sol Energ Mat Sol C, 198-204 (2018).

[3] Philipps, S. P., Bett, A.W., Horowitz, K., Kurtz, S., "Current status of concentrator photovoltaic (CPV) technology," National Renewable Energy Lab, Golden, CO, No. NREL/TP-5J00-65130 (2015).

[4] "National Solar Radiation Data Base: 1991-2005 Update: Typical Meteorological Year 3," NREL. Retrieved 01 July 2019. hhttp:// rredc.nrel.gov/solar/old_data/nsrdb/1991-2005/tmy3/i.

[5] Polman, A., and Atwater, H.A., "Photonic design principles for ultrahigh-efficiency photovoltaics," Nat. Mater. 11(3), 174 (2012).

[6] Russo, J.M., Zhang, D., Gordon, M., Vorndran, S., Wu, Y., and Kostuk, R.K., "Spectrum splitting metrics and effect of filter characteristics on photovoltaic system performance," Opt. Express 22(102), A528-A541 (2014).

[7] McCambridge, J. D., Steiner, M.A., Unger, B.L., Emery, K.A., Christensen, E.L., Wanlass, M.W., Gray, A.L.,"Compact spectrum splitting photovoltaic module with high efficiency." Prog. Photovoltaics 19(3), 352-360 (2011).

[8] Wu, Y., Kostuk, R.K., "Two-junction holographic spectrum-splitting microconcentrating photovoltaic system," J. Photonics Energy 7(1), 017001 (2017).

[9] Vorndran, S.D., Chrysler, B., Wheelwright, B., Angel, R., Holman, Z., Kostuk, R., "Off-axis holographic lens spectrum-splitting photovoltaic system for direct and diffuse solar energy conversion," Appl. Opt. 55(27), 7522-7529 (2016).

[10] Guerrero-Lemus, R., Vega, R., Kim, T., Kimm, A., Shephard, L.E., "Bifacial solar photovoltaics-A technology review," Renew. Sust. Energy Rev. 60, 1533-1549 (2016).

[11] "International Technology Roadmap for Photovoltaic (ITRPV) 2017 Results plus 2018 Maturity Report," ITRPV, Retrieved July 15 2019. https://itrpv.vdma.org/en/ueber-uns

[12] Ayala Pelaez, S., Deline, C., MacAlpine, S.M., Marion, B., Stein, J.S., Kostuk, R.K., "Comparison of bifacial solar irradiance model predictions with field validation," IEEE J. Photovolt. 9(1), 82-88 (2018).

[13] Sun, X., Khan, M.R., Deline, C., Alam, M.A., "Optimization and performance of bifacial solar modules: A global perspective," Appl. Energ. 212, 1601-1610 (2018). 
[14] Castillo-Aguilella, J. E., Hauser, P.S., "Multi-variable bifacial photovoltaic module test results and best-fit annual bifacial energy yield model." IEEE Access 4, 498-506 (2016).

[15] Luque, A., Lorenzo, E., Sala, G., López-Romero, S., "Diffusing reflectors for bifacial photovoltaic panels," Solar cells 13(3), 277-292 (1985).

[16] Wu, Y., Chrysler, B. D., Kostuk, R. K., "Design and fabrication of cascaded dichromate gelatin holographic filters for spectrum-splitting PV systems," J. Photonics Energy 8(1), 017001 (2018).

[17] Chrysler, B. D., Wu, Y., Yu, Z., Kostuk, R.K., "Volume holographic lens spectrum-splitting photovoltaic system for high energy yield with direct and diffuse solar illumination," Proc. SPIE 120368, 103680G (2017).

[18] Stojanoff, C. G., "Review of the technology for the manufacturing of large-format DCG holograms for technical applications," Proc. SPIE 3011, 267-278 (1997).

[19] Moharam, M. G., Gaylord, T. K., "Rigorous coupled-wave analysis of planar-grating diffraction," JOSA 71(7) 811818 (1981).

[20] "FRED Optical Engineering Software," Photon Engineering, Inc. Retrieved 15 July 2019.

https://photonengr.com/fred-software/

[21] Green, M. A., Hishikawa, Y., Dunlop, E. D., Levi, D. H., Hohl-Ebinger, J., Ho-Baillie, A. Y. W., "Solar cell efficiency tables (version 52)," Prog. Photovoltaics 26(7), 427-436 (2018).

[22] Yu, Z. J., Fisher, K.C., Wheelwright, B.M., Angel, R.P., Holman, Z.C., "PVMirror: a new concept for tandem solar cells and hybrid solar converters," IEEE J. Photovolt. 5(6), (2015).

[23] Prism Solar Technologies, Retrieved 15 July 2019. https://www.prismsolar.com/our-products 